\title{
Gerenciamento de Mobilidade em Redes Veiculares de Dados Nomeados Baseado na Estabilidade do Enlace
}

\author{
Antonio M. de Sousa ${ }^{1 *}$, Leobino N. Sampaio ${ }^{1 *}$ (Orientador) \\ ${ }^{1}$ Programa de Pós-Graduação em Ciência da Computação (PGCOMP) \\ Departamento de Ciência da Computação - Universidade Federal da Bahia (UFBA) \\ Salvador - BA - Brasil \\ \{antonio.mateus, leobino\}@ufba.br
}

\begin{abstract}
Vehicle Named Data Networks arise from the union of ad hoc vehicle networks and named data networks, as a way of absorbing the attributes of the information-centric networks paradigm. Despite the significant improvements achieved, old challenges remain. Packet flooding is an example of a challenge that severely impairs the functioning of the network. Therefore, this work proposes strategies that take advantage of native characteristics to selectively forward packets, avoiding flooding. Results obtained from the simulations show a reduction of more than $49 \%$ in the number of retransmissions of interest packages when compared to other strategies found in the literature. Meanwhile, a decrease in retransmission of content packets is around $35 \%$ over the same experiments.
\end{abstract}

Resumo. As Redes Veiculares de Dados Nomeados surgem da união das redes veiculares ad hoc e das redes de dados nomeados, como forma de absorver os atributos do paradigma das redes centradas na informação. Apesar das melhorias significativas obtidas, desafios antigos persistem. A inundação de pacotes é um exemplo de desafio que prejudica severamente o funcionamento da rede. Sendo assim, nesta dissertação são propostas estratégias que usufruem das características nativas para realizar encaminhamento de pacotes de forma seletiva, evitando a inundação. Resultados obtido das simulações mostram uma redução superior a $49 \%$ no número de retransmissões de pacotes de interesses se comparada às demais estratégias encontradas na literatura. Enquanto isso, uma diminuição na retransmissão de pacotes de conteúdo está em torno de $35 \%$ em relação ao mesmos experimentos.

\section{Introdução}

As redes veiculares de dados nomeados (do inglês, Vehicular Named Data Networks VNDN) consistem na união das redes veiculares Ad hoc (do inglês, Vehicular Ad hoc Networks - VANETs), no qual a arquitetura TCP/IP foi substituída pelo paradigma das redes centradas na informação (do inglês, Information-Centric Networking - ICN), mais precisamente sua implementação mais conhecida: as redes de dados nomeados (do inglês, Named Data Networks- NDN). Em VNDN, as VANETs herdam os principais atributos das NDN, isto é, suporte a mobilidade, segurança a nível de pacote, caching nativo na

${ }^{*}$ Os autores agradecem o apoio da FAPESB e da CAPES. 
camada de rede e comunicação orientada ao conteúdo. Por meio dessas características, as VANETs conseguem obter uma melhora significativa ao lidar com os desafios do cenário móvel com suas topologias dinâmicas [Saxena et al. 2016, Araújo et al. 2019a].

Ao herdar as propriedades da arquitetura NDN, a comunicação em VNDNs se baseia em dois pacotes, são eles o pacote de interesse e pacote de dados, usados para requisitar e obter conteúdos, respectivamente. Além disso, os nós utilizam as principais estruturas de dados presentes na comunicação em NDN: tabela de interesses pendentes (do inglês, Pending Interest Table - PIT) e base de conteúdos (do inglês, Content Store - CS). A PIT é responsável por armazenar a lista de interesses pendentes e interesses já satisfeitos, para detectar loops e coletar métricas. A CS consiste na cache que armazena os conteúdos produzidos pelo nó além dos conteúdos recebidos recebidos no processo de encaminhamento [Yaqub et al. 2016]. Apesar da Forwarding Information Base (FIB) fazer parte da arquitetura NDN, a mesma não é utilizada em VNDN, pois a manutenção seria muito complexa devido a dinamicidade do meio e conteúdos de disponibilidade temporária [de Sousa et al. 2018a].

Em VNDN, a principal abordagem adotada na descoberta de conteúdos consiste no envio de pacotes de interesses por difusão (broadcast). Ao adotar esse método de comunicação, contudo, surge o problema do broadcast storm [Ahmed et al. 2015], onde os nós retransmitem sucessivas vezes o mesmo pacote para seus vizinhos até que o nó produtor do conteúdo seja alcançado ou o tempo de vida do interesse expire [Ahmed et al. 2016]. Esse problema resulta em vários subproblemas, tais como, a saturação dos enlaces, lotação das PITs, lentidão, perdas, dentre outros [Araújo et al. 2019a, de Sousa et al. 2018a]. Por esses fatores, o problema de broadcast storm de pacotes de interesse e pacotes de dados em VNDN passa a ser uma questão em aberto e que necessita de maiores investigações [Ahmed et al. 2015].

Com base na discussão apresentada, nesta dissertação é discutida a seguinte questão de pesquisa: Como mitigar a inundação de pacotes em VNDN de maneira simples, adaptativa, sem prejudicar o desempenho da rede além de manter os níveis de satisfação estáveis? Como solução, são introduzidas as estratégias LinkStability-based Interest Forwarding (LSIF) e a NEighborhood STability based forwarding Strategy (NESTS). Ambas as estratégias se aproveitam de uma característica nativa, que também representa umas das maiores dificuldades, das VANETs que é o tempo de vida estimado do enlace (do inglês, Estimated Link Lifetime - ELT). O ELT foi escolhido pois é uma métrica que pode ser obtida independente do ambiente (p.ex: urbano, rural, rodovia), o que torna as estratégias adaptativas e dinâmicas. Apesar de utilizarem o ELT, LSIF e NESTS agem de forma diferente para alcançar seus objetivos. Na LSIF o ELT é calculado em relação ao encaminhador atual que acabou de receber o interesse e o antigo remetente. Dessa forma o nó atual consegue determinar se estará nas redondezas do antigo remetente quando o conteúdo retornar. Já na NESTS, o ELT é empregado para calcular o tempo estimado de vida da vizinhança (do inglês, Neighborhood Estimated LifeTime NELT) de um nó. O NELT é aplicado para avaliar a estabilidade de uma vizinhança para onde um interesse será encaminhado.

Em suma, as contribuições do presente trabalho são: i) duas novas estratégias de encaminhamento de interesses que minimizam os efeitos do broadcast storm de interesses e dados; ii) apresentação dos diferentes usos da métrica do tempo de vida estimado do 
enlace; e iii) avaliação das propostas em ambientes diversos através de simulações conduzidas no simulador NDNSim ${ }^{1}$ [Mastorakis et al. 2015]. Na avaliação experimental, as estratégias foram estressadas para destacar seus pontos fortes bem como suas fraquezas. Para isso foram elencadas variáveis de resposta como interesses retransmitidos, dados retransmitidos, taxa de satisfação de interesses e atraso no recebimento do conteúdo.

\section{Estratégias Propostas}

\subsection{A LSIF}

A estratégia LSIF foi desenvolvida para controlar a propagação indevida de pacotes de interesses através de técnicas simples e adaptativas. Para isso, a LSIF faz uso do tempo de vida estimado do enlace no processo de tomada de decisão de encaminhamento. Este processo ocorre da seguinte forma: um consumidor $C$ interessado em um conteúdo $d$ expressa seu interesse por meio do pacote $i$. O pacote por sua vez é recebido pelo nó $E_{1}$, no comportamento padrão da NDN os nós iriam retransmitir $i$ caso não possuam o dado $d$, no entanto, isso é prejudicial para o funcionamento da rede. Na LSIF, um nó ao receber um pacote de interesse busca pelas informações de velocidade e posição do último encaminhador, no caso, o consumidor inicialmente insere tais informações no campo nome do interesse (p.ex: /prefixo/¡velocidade ${ }_{i} /$ posição $_{i}$ ), aproveitando a estrutura existente. Uma vez encontrada as informações, $E_{1}$ calcula o ELT em relação ao antigo encaminhador e checa se o ELT obtido é superior a um limiar $\alpha$, se sim, $E_{1}$ está apto a realizar o encaminhamento. Caso contrário, o pacote é descartado para evitar retransmissão desnecessária.

\subsubsection{Tempo de vida estimado do enlace}

nesta dissertação é utilizado o tempo estimado de vida do enlace, que consiste no intervalo estimado de tempo (segundos) em que dois veículos permanecerão no raio de transmissão um do outro. Na literatura é possível encontrar diversas formas de se obter o ELT [Boukerche et al. 2017], nesta dissertação é usado os Kinetic Graphs [Härri et al. 2008]. Nessa técnica, os nós precisam compartilhar suas informações de velocidade e posição constantemente, o que não é um problema em VANETs considerando a existência dos beacons. Por outro lado, a LSIF age de forma reativa, onde as informações do nó são transmitidas utilizando o próprio pacote de interesse. É válido mencionar que no cálculo do ELT é assumida a premissa de que os veículos seguem uma trajetória linear por um breve período de tempo, tal abordagem também é utilizada em [Härri et al. 2008] e [Souza et al. 2013].

\subsubsection{Tomada de decisão}

A Figura 1 ilustra a característica reativa da LSIF, em que o nó que recebe o interesse $E_{1}$ é quem realiza a tomada de decisão de encaminhamento. Isto é, $E_{1}$ ao receber o interesse ( $1^{\circ}$ passo), coleta as informações de mobilidade de $C$ e calcula o ELT incluindo também sua velocidade e posição. A partir disso, $E_{1}$ obtém o ELT e a próxima etapa é comparar se esse valor é superior a um limiar $\alpha\left(2^{\circ}\right.$ passo) . O $\alpha$ representa uma medida de confiança (em segundos) que garante que o nó está apto a realizar o encaminhamento. O valor do

\footnotetext{
${ }^{1}$ http: //ndnsim.net/2.3/
} 
limiar pode ser dinamicamente calculado a partir de aferições do próprio ambiente (p.ex: atraso médio), no entanto, para fins de simplicidade, nesta dissertação foi adotado um $\alpha$ fixo, mais precisamente os valores 5s e 10s. Se $E L T<\alpha$ então o pacote é descartado (Drop).

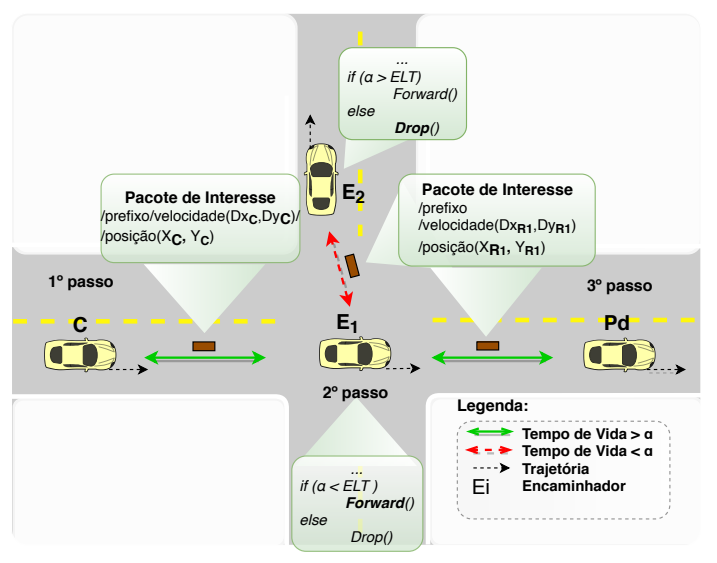

Figura 1. Cenário de VNDN com a adoção da LSIF.

\subsection{A NESTS}

Após o desenvolvimento da LSIF, a mesma foi colocada testada sob cenários diversos para identificar possível pontos de falha e melhorias. Com base nas observações feitas, foi constatado que tanto LSIF tem perda de desempenho quando há mais produtores de um mesmo conteúdo na rede. Isso causa um aumento expressivo no número de retransmissão de pacotes de dados. Tendo em vista este desafio, a solução obtida está na redução do número total de encaminhadores na rede por meio de uma abordagem proativa do nós. Ou seja, o próprio nó é quem decide quem será o próximo encaminhador para um dado interesse.

Utilizando os mesmos princípios da LSIF, a NESTS foi então criada com o intuito de resolver o problema supracitado. No entanto, a NESTS realiza uma tomada de decisão diferenciada na qual os nós mapeiam sua vizinhança com o uso de pacotes de interesse especiais que transmitem as informações sobre o identificador do nó, tamanho da vizinhança do nó (grau), o NELT atual e informações de mobilidade do nó. Essas informações são concatenadas no prefixo (e.g., $/ H E L L O / I D=21 /$ num_vizinhos $=5 / N E L T=10.2 \mathrm{~s} /($ velocidade,posição)) e armazenadas em uma tabela de vizinhos (do inglês, Neighborhood Table - NT). O NELT é uma métrica que determina o tempo de vida estimado de uma vizinhança. Esse valor é calculado por cada nó na vizinhança de acordo com a Equação 1, que é definida pelo somatório do ELT de cada vizinho $j$ do nó $i$ dividido pelo número total de vizinhos $\left|E_{i}\right|$.

$$
N E L T_{i}=\frac{\sum_{j=1}^{\left|E_{i}\right|} \operatorname{ELT}(i, j)}{\left|E_{i}\right|}, \quad \text { onde } j=1 . .\left|E_{i}\right|,
$$

A Figura 2 explicita as etapas citadas em um grafo não-direcionado com os vértices representando os veículos e as arestas suas conexões. Nesse cenário, o vértice $C$ é um consumidor que possui os vizinhos $E_{1}$ e $E_{2}$ em seus respectivos grupos, $G_{1}$ e $G_{2}$. 
O nó $C$ ao eleger um emissor verifica que apesar de $E_{2}$ detém uma maior vizinhança se comparado a $E_{1}$, seu NELT é menor. Ou melhor, a vizinhança de $E_{2}$ está prestes a se desfazer. Logo, $C$ conclui que a escolha mais adequado é o nó $E_{1}$ com uma vizinhança menor mas estável.

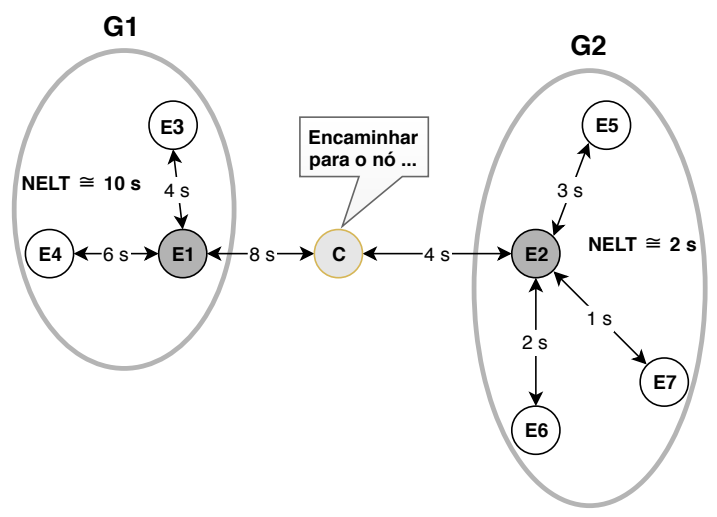

Figura 2. Funcionamento da NESTS.

\section{Experimentos e Resultados}

\subsection{Cenário de Avaliação}

As estratégias foram implementadas e avaliadas no simulador ndnSIM onde as simulações foram divididas em dois cenários, urbano e rodovia. Os experimentos executados seguiram os mesmo comportamento para cada estratégia comparada. Por fim, os dados coletados foram estatisticamente avaliados utilizando um intervalo de confiança com um nível de confiança de $95 \%$.

\subsection{Metodologia de Avaliação}

Na metodologia seguida para avaliar a NESTS, 30\% dos nós desempenharam o papel de consumidores, enquanto a porcentagem de produtores de conteúdo variou de 10, 15, 20 a 25 por cento. Cada simulação foi replicada trinta vezes sempre variando a semente de aleatoriedade com os papéis sendo distribuídos de forma aleatória, como na metodologia seguida por [Boukerche et al. 2017]. A estratégia escolhida para comparação foi a estratégia nativa de NDN, Naive Multicast, CODIE [Ahmed et al. 2016] e a RUFS [Ahmed et al. 2015], que se baseia nas informações coletadas de sua vizinhança para selecionar apenas um encaminhador. O número de nós foi de 25, 50, 75 e 100. As métricas avaliadas foram o ataso na satisfação (Interest Satisfaction Delay - ISD), restransmissões de interesses (Forwarded Interests Packets - FIP), satisfação de interesses (Interests Satisfaction Rate - ISR) e dados retransmitidos (Forwarded Data Packets - FDP).

\subsection{Análise dos Resultados}

A LSIF foi inicialmente comparada com a estratégia CODIE e Naive Multicast. Entretanto, para fins de simplicidade é abordado a comparação com a CODIE, como estratégia base. Na Tabela 1, são sumarizados ganhos (+) e perdas (-) do cenário urbano que evidencia que os ganhos da LSIF, comparado a estratégia Multicast Nativo, no quesito redução de retransmissões e tempo de resposta superam a queda no ISR mesmo em um cenário de 
alta mobilidade. A CODIE, por sua vez, reduziu bastante o atraso no tempo de satisfação dos interesses dado que o pacote de conteúdos possui vários caminhos disponíveis para retornar ao consumidor por causa da inundação na fase de requisição.

Tabela 1. Desempenho da LSIF comparado a estratégia CODIE em cenário urbano [de Sousa et al. 2018a].

\begin{tabular}{|l|l|l|l|l|}
\cline { 2 - 5 } \multicolumn{1}{c|}{} & FIP & FDP & ISR & ISD \\
\hline LSIF $_{\alpha=5}$ & $+49,24 \%$ & $+31,19 \%$ & $-16,91 \%$ & $-20,98 \%$ \\
\hline LSIF $_{\alpha=10}$ & $+51,61 \%$ & $+44,24 \%$ & $-18,26 \%$ & $-13,60 \%$ \\
\hline
\end{tabular}

Já na Tabela 2, é retratado o resumo do desempenho da LSIF. Devido a simplicidade do cenário de rodovia em termos de mobilidade, o número de retransmissões é maior. Apesar disso, a LSIF consegue manter tanto FIP quanto FDP abaixo da metade do total retornado pela CODIE. Além disso, os níveis de ISR e ISD tendem a se manter estáveis. Tal queda no ISR e ISD representam um tradeoff a ser abordado no futuro.

Tabela 2. Desempenho da LSIF comparado a estratégia CODIE em rodovia [de Sousa et al. 2018a].

\begin{tabular}{|l|l|l|l|l|}
\cline { 2 - 5 } \multicolumn{1}{c|}{} & FIP & FDP & ISR & ISD \\
\hline LSIF $_{\alpha=5}$ & $+49,733 \%$ & $-19,998 \%$ & $-35,937 \%$ & $-28,628 \%$ \\
\hline LSIF $_{\alpha=10}$ & $+55,099 \%$ & $+26,279 \%$ & $-39,10 \%$ & $+16,70 \%$ \\
\hline
\end{tabular}

Nesta segunda etapa da análise é mostrado os resultados da NESTS. A Figura 3 sumariza a avaliação da NESTS frente às demais estratégias. Os resultados consistem na variação percentual média representando os ganhos da NESTS $(+)$ e suas perdas $(-)$ agrupados nos cenários urbano (i.e., Figura 3(a)) e rodovia (i.e., Figura 3(b)). Ao observar os resultados extraídos do cenário urbano na Figura 3(a), nota-se uma redução constante no número de retransmissões de interesses e de dados. Como consequência há perdas que se refletem no ISD e ISR, destacando um tradeoff da proposta. Novamente, o objetivo da NESTS é mitigar a inundação de pacotes enquanto mantém os níveis de satisfação aceitáveis.

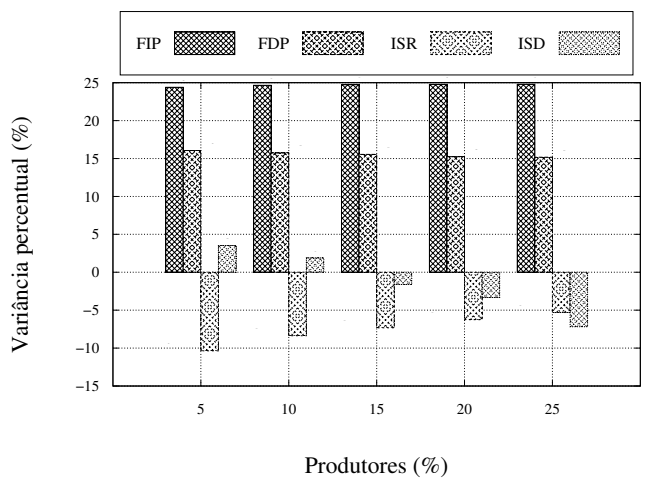

(a) Avaliação no cenário urbano.

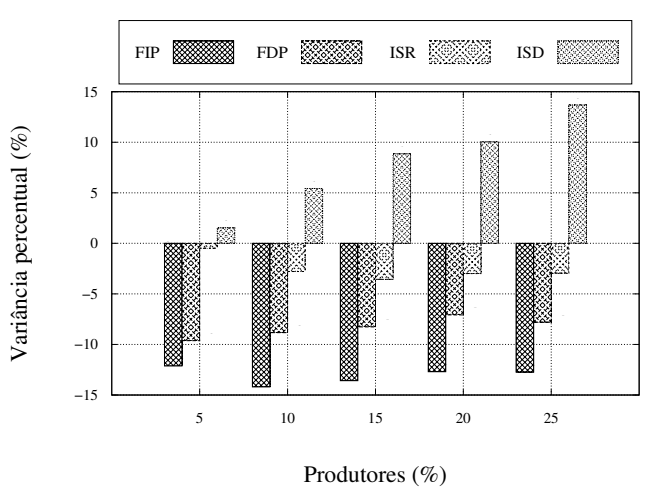

(b) Avaliação no cenário de rodovia.

Figura 3. Variação percentual da NESTS comparada a LSIF. 
No cenário de rodovia representado na Figura 3(b), é visto que a NESTS teve uma performance inferior à RUFS. Isso ocorre porque no ambiente de rodovia, os nós tendem a ficarem mais próximos dificultando a tomada de decisão e elevando o número de timeouts, ou seja, estouro de temporizadores. Os resultados destacados comprovam que a NESTS consegue reduzir tanto FIP quanto FDP mesmo com uma maior porcentagem de produtores ativos na rede. Ademais, também é perceptível que com o aumento no número de produtores as perdas relacionadas ao ISR e ISD são atenuadas em ambos os ambientes observados.

\section{Conclusão}

A NDN por mais robusta que possa ser ainda é uma arquitetura em desenvolvimento que requisita melhorias e adaptações de acordo com o ambiente de implantação. Nesta dissertação foram apresentadas as estratégias LSIF e NESTS que abordam o desafio do broadcast storm de pacotes em VNDN. Os resultados obtidos revelam que o objetivo das estratégias foram alcançados, isto é, a LSIF conseguiu mitigar a inundação de interesses através de seu encaminhamento seletivo e da mesma forma a NESTS diminuiu o número de cópia de dados retransmitidos na rede. Vale ressaltar que poucas alterações foram feitas na NDN para possibilitar o funcionamento das propostas.

\section{Publicações}

Os resultados alcançados ao longo desta pesquisa foram divulgados através das seguintes publicações científicas:

- Stability-Based Interest-Forwarding Strategy For Vehicular Named Data Networks [de Sousa et al. 2018a] - IEEE Internet Computing, 2018.

- Encaminhamento Seletivo de Interesses em Redes Veiculares de Dados Nomeados Baseado no Tempo de Vida do Enlace [de Sousa et al. 2018b] - SBRC, 2018.

Demais publicações relacionadas:

- Uma Estratégia de Encaminhamento Eficiente para Redes de Veículos Aéreos não Tripulados de Dados Nomeados [Araújo et al. 2019b] - SBRC, 2019.

- SCaN-Mob: An opportunistic caching strategy to support producer mobility in named data wireless networking [Araújo et al. 2019a] - IEEE Computer Networks, 2019.

- On Evaluating the Influence of User's Music Listening Habits on Cache Replacement Policies [Pires et al. 2018]- IEEE ISCC, 2018.

- Armazenamento Oportunista em Redes de Dados Nomeados Sem Fio como Suporte à Mobilidade de Produtores [Araújo et al. 2018] SBRC, 2018.

Por fim, a submissão da NESTS foi realizada na conferência IEEE WoWMoM 2020, após rejeição na fase de major revision na IEEE Communications Letters.

\section{Referências}

Ahmed, S. H., Bouk, S. H., and Kim, D. (2015). RUFS: RobUst forwarder selection in vehicular content-centric networks. IEEE Communications Letters, 19(9):1616-1619.

Ahmed, S. H., Bouk, S. H., Yaqub, M. A., Kim, D., Song, H., and Lloret, J. (2016). CODIE: Controlled data and interest evaluation in vehicular named data networks. IEEE Transactions on Vehicular Technology, 65(6):3954-3963. 
Araújo, F. R. C., de Sousa, A. M., and Sampaio, L. N. (2018). Armazenamento oportunista em redes de dados nomeados sem fio como suporte a mobilidade de produtores. Simpósio Brasileiro de Redes de Computadores e Sistemas Distribuídos (SBRC), 36.

Araújo, F. R. C., de Sousa, A. M., and Sampaio, L. N. (2019a). Scan-mob: An opportunistic caching strategy to support producer mobility in named data wireless networking. Computer Networks, 156:62-74.

Araújo, F. R. C., de Sousa, A. M., and Sampaio, L. N. (2019b). Uma estratégia de encaminhamento eficiente para redes de veículos aéreos não tripulados de dados nomeados. Simpósio Brasileiro de Redes de Computadores e Sistemas Distribuídos (SBRC), 37:895-908.

Boukerche, A., Coutinho, R. W., and Yu, X. (2017). Lisic: A link stability-based protocol for vehicular information-centric networks. In 2017 IEEE 14th International Conference on Mobile Ad Hoc and Sensor Systems (MASS), pages 233-240. IEEE.

de Sousa, A. M., Araújo, F. R. C., and Sampaio, L. N. (2018a). A link-stability-based interest-forwarding strategy for vehicular named data networks. IEEE Internet Computing, 22(3):16-26.

de Sousa, A. M., RC, F. A., and Sampaio, L. N. (2018b). Encaminhamento seletivo de interesses em redes veiculares de dados nomeados baseado no tempo de vida do enlace. In Simpósio Brasileiro de Redes de Computadores (SBRC), volume 36.

Härri, J., Bonnet, C., and Filali, F. (2008). Kinetic mobility management applied to vehicular ad hoc network protocols. Computer Communications, 31(12):2907-2924.

Mastorakis, S., Afanasyev, A., Moiseenko, I., and Zhang, L. (2015). ndnsim 2.0: A new version of the ndn simulator for ns-3. NDN, Technical Report NDN-0028.

Pires, S. S., Ribeiro, A. V., de Sousa, A. M., Freitas, A. E., and Sampaio, L. N. (2018). On evaluating the influence of user's music listening habits on cache replacement policies. In 2018 IEEE Symposium on Computers and Communications (ISCC), pages 0093000933. IEEE.

Saxena, D., Raychoudhury, V., Suri, N., Becker, C., and Cao, J. (2016). Named data networking: a survey. Computer Science Review, 19:15-55.

Souza, A. B., Celestino, J., Xavier, F. A., Oliveira, F. D., Patel, A., and Latifi, M. (2013). Stable multicast trees based on ant colony optimization for vehicular ad hoc networks. In The International Conference on Information Networking 2013 (ICOIN), pages 101-106. IEEE.

Yaqub, M. A., Ahmed, S. H., Bouk, S. H., and Kim, D. (2016). Interest forwarding in vehicular information centric networks: a survey. In Proceedings of the 31st Annual ACM Symposium on Applied Computing, pages 724-729. ACM. 